\title{
BMJ Open Efficacy and safety of the Shexiang Baoxin Pill for the treatment of coronary artery disease not amenable to revascularisation: study protocol for a randomised, placebo-controlled, double- blinded trial
}

Pan-pan Tian, ${ }^{1,2}$ Jun Li, ${ }^{1}$ Jian Gao, ${ }^{1,2}$ Ying Li ${ }^{1,2}$

To cite: Tian P, Li J, Gao J, et al. Efficacy and safety of the Shexiang Baoxin Pill for the treatment of coronary artery disease not amenable to revascularisation: study protocol for a randomised, placebo-controlled, doubleblinded trial. BMJ Open 2018;8:e018052. doi:10.1136/ bmjopen-2017-018052

- Prepublication history for this paper is available online To view these files, please visit the journal online (http://dx.doi org/10.1136/bmjopen-2017018052).

Received 7 June 2017 Revised 26 November 2017 Accepted 30 November 2017

Check for updates

${ }^{1}$ Guang'anmen Hospital, China Academy of Chinese Medical Sciences, Beijing, China ${ }^{2}$ Graduate School Department, Beijing University of Chinese Medicine, Beijing, China

Correspondence to Mr Jun Li; gamyylj@163.com

\section{ABSTRACT}

Introduction Coronary artery disease (CAD) not amenable to revascularisation indicates that the coronary arteries have severe diffuse lesions or calcifications, or that CAD is complicated with severe multiple-organ disease. Currently, Western medicines available for the treatment of CAD not amenable to revascularisation are limited. Shexiang Baoxin Pill (SBP), a type of Chinese patent medicine, has been widely used to treat CAD in China for many years. Previous studies have shown that long-term administration of SBP (1-2 pills three times daily, for at least 6 months) for treatment of CAD is effective and safe, with a significant, long-term effect. This study aims to evaluate the efficacy and safety of SBP in patients with CAD not amenable to revascularisation.

Methods and analysis This is a multicentre, randomised, double-blinded, placebo-controlled clinical trial. A total of 440 participants will be randomly allocated to two groups: the intervention group and the placebo group. Based on conventional treatment with Western medicine, the intervention group will be treated with SBP and the placebo group will be treated with SBP placebo. The primary outcomes include major adverse cardiovascular events (including angina, acute myocardial infarction, pulmonary embolism and aortic dissection). The secondary outcomes include $C$ reactive protein, $B$-type natriuretic peptide, ECG, echocardiographic parameters (ejection fraction percentage and the E/A ratio) and hospital readmission rates due to CAD. Assessments will be performed at baseline (before randomisation) and at 24 weeks after randomisation.

Ethics and dissemination The protocol has been approved by the Research Ethics Committee of Guang'anmen Hospital, China Academy of Chinese Medical Sciences in Beijing, China (reference: 2016-129-KY-01). The results of this study will be published in a peerreviewed journal and will be used as a basis for a multisite trial.

Trial registration number NCT03072121; Pre-results.

\section{BACKGROUND}

Coronary artery disease (CAD) is characterised by the narrowing or blockage of arteries and vessels that provide oxygen and blood to the heart. It is the leading cause among the 235 causes of death in human; CAD kills more than 7 million people annually worldwide and is predicted to remain the top cause of death for the next 20 years. ${ }^{1}$ CAD not amenable to revascularisation mainly refers to severe diffuse left main coronary artery and three-vessel stenosis, calcifications or lesions, or CAD complicated with severe multiple-organ disease, such as severe heart failure, infection, blood diseases, cancer cachexia, lung dysfunction or renal insufficiency. ${ }^{2}$ With the rapid increase in patients with diabetes and obesity, patients with CAD not amenable to revascularisation are expected to increase exponentially. ${ }^{3}$ Currently, the main treatment methods for CAD include lifestyle changes, medical treatment (cholesterol-lowering medications, beta-blockers, nitroglycerin, calcium antagonists and so on), coronary interventions and surgery. ${ }^{4-6}$ Among these treatments, coronary revascularisation, including percutaneous coronary intervention and coronary artery bypass surgery, is an advanced and effective therapeutic method for CAD, especially acute coronary syndrome. However, in a contemporary series of patients undergoing coronary angiography, $28.8 \%$ had significant CAD and did not undergo complete revascularisation, of whom $12.8 \%$ was partially revascularised, $9.3 \%$ was managed medically and $6.7 \%$ had 'no-options'; these patients exhibited higher mortality at 3 years compared with completely revascularised patients. ${ }^{7}$ Even Medicare has 
no classification code for patients with CAD not amenable to revascularisation, and the US Task Force addressing this situation stresses the urgent need for studies. ${ }^{8}$

Chinese herbal medicine (CHM), a popular type of complementary medicine, plays an important role in treating CAD in China. According to the theory of traditional Chinese medicine (TCM), all the related symptoms and signs at a certain phase of a disease are generalised as a syndrome ('Zheng' in Chinese medicine), which is the basic unit and key concept of TCM. ${ }^{9}$ Patients with CAD can be divided into different syndromes. In the diagnosis of CAD, ' $Q i$ deficiency and blood stasis syndrome' is an important type diagnosed from the viewpoint of TCM and our previous clinical practice. ${ }^{10}$ Therefore, the principle of 'tonifying $Q i$ and activating blood' is applied in the treatment of CAD not amenable to revascularisation. Shexiang Baoxin Pill (SBP) is based on the Suhexiang pill documented in the Prescription of Peaceful Benevolent Dispensary (Taiping Huimin Hejiju Fang) from the Song Dynasty. It consists of seven herbal medicines, namely musk (Moschus, She Xiang), ginseng root (Radix Ginseng, Ren Shen), cow bezoar (Calculus Bovis, Niu Huang), storax (Styrax, Su He Xiang), cassia bark (Cortex Cinnamomi, Rou Gui), toad venom (Venenum Bufonis, Chan Su) and borneol (Borneolum Syntheticum, Bing Pian). It has been widely used for the treatment of CAD in China for many years. Modern studies have indicated that the major pharmacological mechanisms of SBP include improving endothelial cell function, inhibiting vascular inflammation through decreasing the level of $\mathrm{C}$ reactive protein and plasma homocysteine, stabilising atherosclerotic plaque, promoting therapeutic angiogenesis, inhibiting the abnormal proliferation of vascular smooth muscle cells, reversing myocardial fibrosis, dilating coronary arteries, improving myocardial ischaemia and reducing myocardial infarct size. ${ }^{11-14}$ Experimental studies have shown that SBP is quickly absorbed in the body, is quickly eliminated and has a short duration of action. Although toad venom (Venenum Bufonis, $\mathrm{Chan} \mathrm{Su}$ ) is toxic when administered alone, its toxicity may be reduced by extending the peak time of toad steroid ingredients via other compatible ingredients in SBP, thus showing the scientific nature of compound compatibility. ${ }^{15}$ In addition, clinical studies have found that long-term SBP administration could reduce the occurrence of angina pectoris events and several other clinical events and reduce the dosage of nitrates used in patients with stable angina pectoris. ${ }^{16-19}$ In addition, the adverse reactions to SBP are mild, and studies have not shown that SBP is harmful to liver or kidney function. ${ }^{19} 20$ However, whether SBP is effective for the treatment of CAD not amenable to revascularisation is still unknown. The aim of this multicentre, randomised, double-blind, placebo-controlled trial is to evaluate the efficacy and safety of SBP in patients with CAD not amenable to revascularisation.

\section{METHODS/DESIGN}

\section{Trial organisation}

The funders will not participate in the study design, data collection, analyses and interpretation, and manuscript preparation. An independent data and safety monitoring board will monitor the conduct and safety of the trial to ensure patient safety. Stopping guidelines and monitoring practices have been established.

\section{Study population}

A total of 440 patients will be recruited from seven centres: Wangjing Hospital, China Academy of Chinese Medical Sciences, Beijing Anzhen Hospital, The Second Affiliated Hospital of Henan University of Traditional Chinese Medicine, Guangdong Provincial Hospital of Traditional Chinese Medicine, Shandong University of Traditional Chinese Medicine, and Shaanxi Hospital of Traditional Chinese Medicine. The trial began in June 2017 and will continue until February 2019.

\section{Recruitment of participants}

Two strategies are being used to recruit patients with CAD not amenable to revascularisation. First, we are displaying recruitment posters outside the clinics. The posters contain brief introductions about the population required, the medicine offered to eligible participants and the contact information of the researcher. Second, we are recruiting participants in outpatient clinics from Wangjing Hospital, China Academy of Chinese Medical Sciences, Beijing Anzhen Hospital, The Second Affiliated Hospital of Henan University of Traditional Chinese Medicine, Guangdong Provincial Hospital of Traditional Chinese Medicine, Shandong University of Traditional Chinese Medicine, and Shaanxi Hospital of Traditional Chinese Medicine. Patients who meet the study criteria are requested to sign a written informed consent form. The consent form includes the nature, objectives, and potential benefits and consequences of the study. Additionally, the consent details the required length of follow-up, supportive care, the name of the principal investigator (JL) responsible for the protocol, and the patient's right to accept or refuse treatment and to terminate participation and withdraw from the study.

\section{Inclusion criteria}

Participants will be included if they met the following requirements: between 55 and 75 years old, diagnosed with severe CAD through coronary arteriography, which showed that the left main coronary artery and three vessels have severe diffuse stenosis, calcification or vascular ectasia, and have ' $Q i$ deficiency and blood stasis syndrome' according to TCM. For the Western medicine diagnostic criteria, we refer to 'Guidelines for the Prevention and Management of Chronic Stable Angina in China'21 (2007 edition), 'Guidelines for the Diagnosis and Management of Non-ST-segment Elevation Myocardial Infarction Acute Coronary Syndromes ${ }^{22}$ (2012 edition), and 'Guidelines for the Diagnosis and 
Management of Acute ST-segment Elevation Myocardial Infarction, ${ }^{23}$ (2010 edition), which were published by the Cardiology Branch of the Chinese Medical Association. In addition, for the TCM diagnostic criteria, we refer to 'Guideline of Clinical Research of New Drugs of Traditional Chinese Medicine.Chest Obstruction,24 (2002 edition). Finally, both cardiologists and cardiac surgeons will decide whether patients with $\mathrm{CAD}$ are in accordance with the inclusion criteria.

\section{Exclusion criteria}

The exclusion criteria are as follows: (1) patients with severe valvular disease or congenital decompensated cardiomyopathy; (2) patients with CAD complicated with severe multiple-organ disease, such as severe heart failure, severe lung, liver or renal dysfunction, peptic ulcer in the active stage, or intracranial haemorrhage; (3) patients who use high-dose steroids due to connective tissue disease; (4) patients with serious infections; (5) patients with malignant tumours; (6) patients with haematopoietic diseases; (7) female patients who are pregnant or lactating; and (8) patients who are eligible for revascularisation.

\section{Handling of withdrawal and data management}

Participants may withdraw from the study at any time for any reason. If any patients want to withdraw, clinicians should ask whether they would be willing to complete the assessments according to the study schedule and record the last day of medicine intake. Patients lost to follow-up and patients who withdraw from the study will be recorded and reported. The data collected in this trial comprise information recorded on case report forms. After every visit at each centre, the data will be entered using the double-entry method.

\section{Interventions}

In addition to conventional Western medical treatment, including antiplatelet, lipid-lowering, antihypertensive or antidiabetic therapy, eligible patients will be allocated to receive SBP or placebo two pills three times daily for 24 successive weeks. The SBP and placebo were produced and packed in a single batch (production batch number: 160299) by Shanghai Hutchison Pharmaceuticals in Shanghai, China. The results of drug quality tests were consistent with the Chinese Medicine Standards of the State Food and Drug Administration. SBP is a compound prepared with Chinese herbs, and their main components are shown in table 1 . The placebo pills have an identical appearance and scent as the active treatment pills. Patients will take the pills orally three times daily for 24 successive weeks. Intake of any other Chinese herbal decoction or Chinese patent medicine for treating CAD is prohibited during the study.

\section{Randomisation and blinding}

Randomisation was performed by an independent statistician. The randomisation sequence (blocked and stratified for centres) was generated using SAS V.9.4 software. Each centre received consecutively coded drugs. All of the drugs provided by the pharmaceutical company are labelled according to the randomisation schedule. This trial is a double-blind trial. The first level is for the case number corresponding to groups (group A and group B), and the second level is for the group corresponding to the intervention (the intervention and placebo groups). The numbers are kept in opaque sealed envelopes. The two levels of blinding are sealed separately and given to the lead clinical researcher. Emergency letters have been sent to each of the centres, are kept with the test drug and will be properly preserved until the end of the trial.

\begin{tabular}{|c|c|c|}
\hline Chinese name & Description & Comments \\
\hline She Xiang & $\begin{array}{l}\text { A substance with a penetrating odour that } \\
\text { is obtained from a gland of the male musk } \\
\text { deer }\end{array}$ & $\begin{array}{l}\text { The original material is banned from use due to the endangered } \\
\text { status of the musk deer; a synthetic compound (muscone) is } \\
\text { used in its place. }\end{array}$ \\
\hline Ren Shen & Root of Chinese ginseng & This is an extract that specifically includes ginsensosides. \\
\hline Su He Xiang & $\begin{array}{l}\text { An aromatic extract of the Liquidambar tree } \\
\text { (Styrax; storax) }\end{array}$ & $\begin{array}{l}\text { It has antiplatelet aggregation, antithrombosis, antimyocardial } \\
\text { ischaemia and other effects. }\end{array}$ \\
\hline Rou Gui & Bark of the cinnamon & $\begin{array}{l}\text { Cinnamon aqueous solution is rich in antioxidants and can } \\
\text { reduce the risk of heart disease and diabetes. }\end{array}$ \\
\hline Bing Pian & $\begin{array}{l}\text { Crystalline aromatic component of certain } \\
\text { plants that predominantly contain borneol. }\end{array}$ & $\begin{array}{l}\text { Due to high cost, the patent medicine contains synthetic } \\
\text { borneol; borneol is known as a cardiac stimulant. }\end{array}$ \\
\hline
\end{tabular}




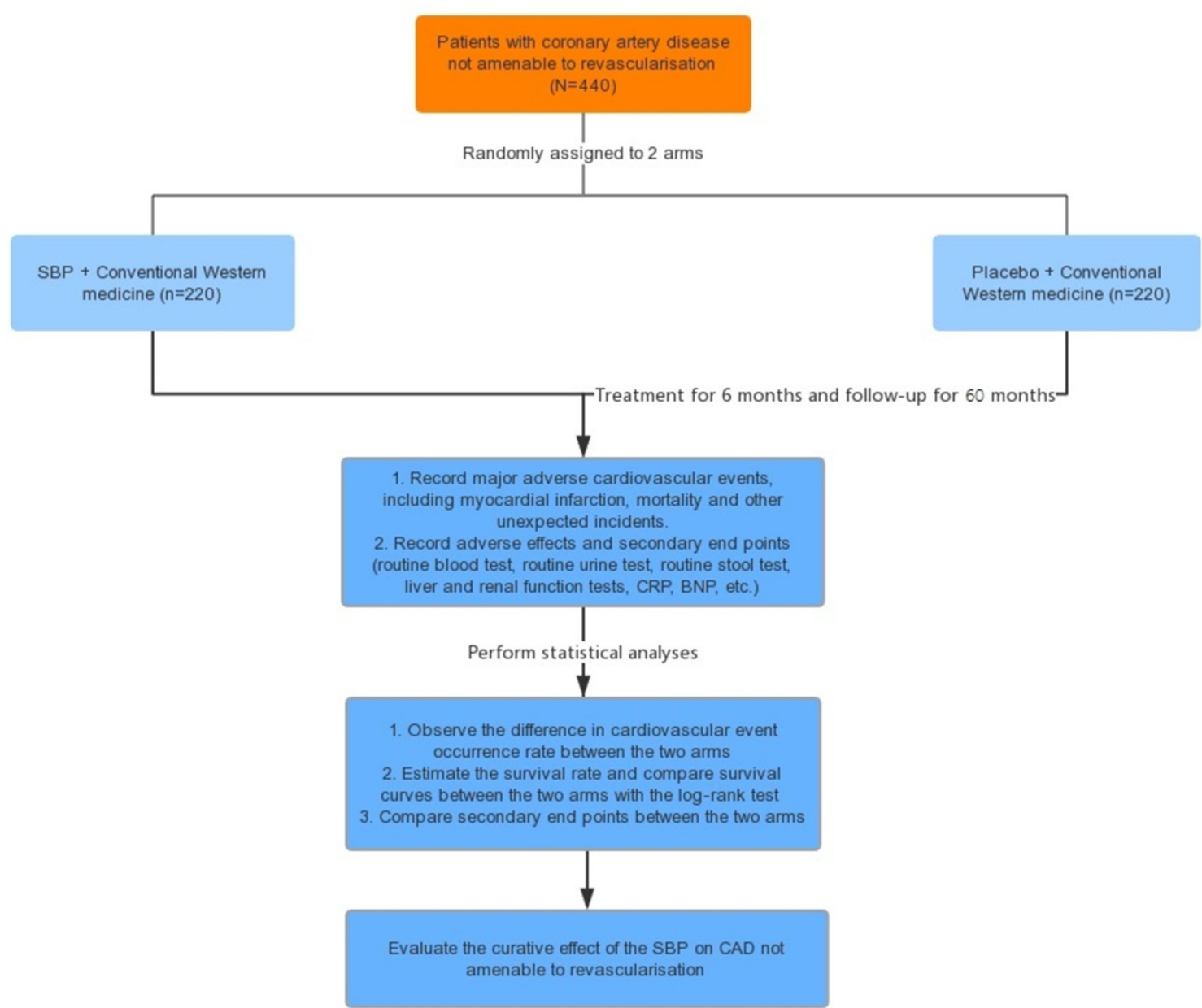

Figure 1 Flow chart. BNP, B-type natriuretic peptide; CAD, coronary artery disease; CRP, C reactive protein; SBP, Shexiang Baoxin Pill.

Treatment assignments will not be revealed to the patients and investigators (including statisticians) until the entire study is completed. The time points are shown in figure 1.

\section{Primary outcomes}

The primary outcomes include mortality and major adverse cardiovascular events (including angina, acute myocardial infarction, pulmonary embolism and aortic dissection).

\section{Secondary outcomes}

The secondary outcomes include C reactive protein, B-type natriuretic peptide, ECG (abnormal ST-T changes), echocardiographic parameters (ejection fraction (EF)\% and the $\mathrm{E} / \mathrm{A}$ ratio) and hospital readmission rate due to $\mathrm{CAD}$.

\section{Safety outcomes}

Safety outcomes include the following: (1) measurement of vital signs, including temperature, blood pressure, respiration and heart rate; (2) routine blood tests, routine urine tests and routine stool tests; (3) blood lipid tests and blood glucose tests; (4) liver function tests (Alanine aminotransferase (ALT), aspartate aminotransferase (AST), $\gamma$-gamma glutamyl transferase $(\gamma$-GT), alkaline phosphatase (ALP) and total bilirubin (TBIL)) and renal function tests (blood urea nitrogen (BUN) and creatinine (Cr)); (5) ECG (mainly ST-T changes); and (6) records of adverse events at any time.

These biological indicators are monitored from the grouping of these patients until the end of follow-up.

\section{Adverse events}

Any unexpected symptoms, vital signs or sicknesses, as long as they cause discomfort, will be recorded as an adverse event. The start date, end date, degree, relationship with the trial medicine and whether the patients drop out of the study will be recorded. Severe adverse events are required to be reported to the lead researcher of the trial, ethics committees and sponsors within 24 hours, and the participants will be provided with every necessary treatment. If the adverse event persists, follow-up will continue until the adverse event disappears.

\section{Sample size}

The incidence of major adverse cardiovascular events will be compared between the groups. The sample size was calculated using the concept of efficiency as presented by Xie. ${ }^{25}$ The efficiency is $80 \%$ in the intervention group and $60 \%$ in the placebo group, as suggested by a previous 
study. The following formula was used for a two-group trial:

$$
n=\left(U_{\alpha}+U_{\beta}\right)^{2} 2 P(1-P) /\left(P_{1}-P_{0}\right)
$$

Based on $\alpha=0.05$ and $\beta=0.2$, the required sample size per group is approximately 90 participants. Allowing for $15 \%$ attrition, we should recruit 208 participants, with 104 patients in each group. Data analysis will be conducted by statisticians who are independent from the research team. An intent-to-treat analysis will be carried out for patients who have received treatment at least once. Missing data will be adjusted using the last observation carried forward method. Per-protocol analysis will be restricted to participants who strictly follow the protocol and complete the study. The database will be maintained using Excel software. The raw data will be independently typed into computers by two statisticians. Every analysis will be conducted using SPSS V.26.0 software. We will calculate the frequency and percentage of variables with the Descriptive statistics program. Pearson's $\chi^{2}$ test will be performed on categorical variables, and Student's t-test will be performed on measurement variables. The log-rank test will be used to assess the difference in survival distributions between the two groups with respect to some failure time outcome.

\section{Ethics and dissemination}

Final results of the trial will be disseminated via publication and ClinicalTrials.gov. Authorship will be determined based on the BMJ Open guidelines.

\section{DISCUSSION}

Antiplatelet therapy is the cornerstone treatment for CAD. It is very important in the prevention of acute or subacute thrombosis and severe cardiovascular events. Currently, aspirin and clopidogrel are the most commonly used antiplatelet drugs. Aspirin plays an important role in the acute phase, as well as the primary and secondary prevention of CAD. Clopidogrel is safe and effective in reducing acute coronary syndrome and ischaemic events in percutaneous coronary intervention patients. In addition, dual therapy with aspirin and clopidogrel has emerged as the gold standard therapy for patients treated with drugeluting stents. However, there is variability in patient responses to antiplatelet therapy. Studies have identified that among patients with $\mathrm{CAD}, 5 \% \sim 45 \%$ are aspirin-resistant, $4 \% \sim 30 \%$ are clopidogrel-resistant and $10 \%$ are resistant to both. ${ }^{26}$ In addition, medication compliance among patients is another problem. Middle-aged and elderly patients are at high risk for CAD. Elderly patients with $\mathrm{CAD}$ are always comorbid with hypertension, diabetes, hyperlipidaemia, cerebral infarction, obstructive pulmonary disease and others. Lifelong medication is necessary to control the development of diseases among this patient population. However, more than $60 \%$ of elderly patients fail to adhere to their medication regimen due to an abundance of drugs but insufficient relief of symptoms. ${ }^{27}$
Therefore, it is necessary to identify other safe and effective treatment methods that can alleviate symptoms and improve the quality of life of patients with CAD.

As a complementary medicine, CHM is attracting attention. ${ }^{28} 29$ Many TCM studies have implied that either single TCM or compound preparations can have multiple target effects in the prevention and treatment of cardiovascular disease. ${ }^{30} 31$ The composition of Chinese herbal compounds is more complicated. They can intervene at each phase of disease occurrence and development. The multiple target effects reflect the advantages of traditional Chinese herb medicine. ${ }^{32} 33$

Treatment with syndrome differentiation is one of the characteristics of the TCM system. Qi deficiency and blood stasis syndrome is the core pathogenesis of CAD. ${ }^{345}$ SBP has the effect of supplementing Qi and activating blood, which is consistent with the core pathogenesis. Numerous studies have identified the efficacy and safety of SBP in treating CAD. However, among these studies, most are based on clinical experience, case reports, case series and expert opinions, whereas large-sample, retrospective, randomised controlled studies are scarce ${ }^{36}$ Whether SBP is effective for the treatment of CAD not amenable to revascularisation still requires confirmation by evidencebased medical research through large-sample, multicentre, randomised controlled clinical trials. To ensure appropriate high-quality methodology and strict quality control, this protocol has been developed according to the the Standard Protocol Items: Recommendations for Interventional Trials 2013, ${ }^{37}$ and the new extension of the Consolidated Standards of Reporting Trials statement. ${ }^{38}$ This study has a randomised, double-blind, parallel, controlled, multiple-centre clinical study design. It may be significant for the improvement of patient prognosis and quality of life.

There are also some limitations to the study that should be considered. Due to restrictions in research project funds and trial period, the treatment duration will be short, and thus additional randomised controlled trials with long-term follow-up are warranted to determine the efficacy and safety of SBP.

Contributors $\mathrm{JL}$ is the principal investigator of this study. PT wrote the first draft of the manuscript. JL was involved in programme design and modifying the articles. YL and JG contributed to the statistical acquisition and analysis of data. All authors critically revised the protocol for important intellectual content and approved the final manuscript.

Funding Funding provided by the Fundamental Research Funds for the Central Public Welfare Research Institutes.

Competing interests None declared.

Patient consent Obtained.

Ethics approval The study was approved by the Research Ethics Committee of Guang'anmen Hospital of the China Academy of Chinese Medical Sciences in Beijing, China (reference: 2016-129-KY-01).

Provenance and peer review Not commissioned; externally peer reviewed.

Open Access This is an Open Access article distributed in accordance with the Creative Commons Attribution Non Commercial (CC BY-NC 4.0) license, which permits others to distribute, remix, adapt, build upon this work non-commercially, and license their derivative works on different terms, provided the original work is 
properly cited and the use is non-commercial. See: http://creativecommons.org/ licenses/by-nc/4.0/

(C) Article author(s) (or their employer(s) unless otherwise stated in the text of the article) 2018. All rights reserved. No commercial use is permitted unless otherwise expressly granted.

\section{REFERENCES}

1. Zhao D. Why dentists need to learn the epidemiological status and prevention strategy of coronary heart disease in China. Chin $J$ Stomatol 2016:51:385-6.

2. Mannheimer C, Camici P, Chester MR, et al. The problem of chronic refractory angina; report from the ESC Joint Study Group on the Treatment of Refractory Angina. Eur Heart J 2002;23:355-70.

3. Gupta S, Pressman GS, Morris DL, et al. Distribution of left ventricular ejection fraction in angina patients with severe coronary artery disease not amenable to revascularization. Coron Artery Dis 2010;21:278-80.

4. Luo $L$. The research progress of treatment methods for coronary heart disease. Pub Med Forum Mag 2013;17:3363-6.

5. Zhang Y, Tang HQ, Li J. Meta-analysis on curative effect and safety of Shexiang Baoxin Wan in treatment of coronary heart diseaseChinese. Chin J Evid Based Cardiovasc Med 2012;4:13-17.

6. Sun H, Cui L. Optimal treatment of multivessel complex coronary artery disease. Exp Ther Med 2014;7:1563-7.

7. Williams B, Menon M, Satran D, et al. Patients with coronary artery disease not amenable to traditional revascularization: Prevalence and 3-year mortality. Catheter Cardiovasc Interv 2010;119:881-91.

8. Lozano I, Capin E, de la Hera JM, et al. Diffuse Coronary Artery Disease Not Amenable to Revascularization: Long-term Prognosis. Rev Esp Cardiol 2015;68:631-3.

9. Jiang M, Zhang C, Zheng G, et al. Traditional chinese medicine zheng in the era of evidence-based medicine: a literature analysis. Evid Based Complement Alternat Med 2012;2012:1-9.

10. Bi YF, Mao JY, Wang XL, et al. Clinical epidemiology survey of the traditional Chinese medicine etiology and syndrome differentiation of coronary artery disease: study protocol of a multicenter trial. Zhong Xi Yi Jie He Xue Bao 2012;10:619-27.

11. Wy L, Shen JP. Research on mechanism of Shexiang Baoxin Pill in the treatment of coronary heart disease. J Emerg Trad Chin Med 2011;20:114-5.

12. Liu Q, Lv C, Zhang WD, et al. Research progress of Shexiang Baoxin Pill. Chin Trad Herb Drugs 2016;47:1409-17.

13. Zhang XZ, Hou YM, Ou ZH. [Effect of Shexiang Baoxin pill on coronary vasodilation by analysis of coronary angiography]. Zhongguo Zhong Xi Yi Jie He Za Zhi 2014;34:1432-5.

14. ZI C, Gu N. Mechanism research of Shexiang Baoxin pill on treating coronary artery disease. Jilin J Trad Chin Med 2011;31:262-3.

15. Liu Q, Lv C, Zhang WD, et al. Advance in Modern Studies on Shexiang Baoxin Pill. China Tradit Herb Drugs 2016;47:1409-17.

16. Zhu H, Luo XP, Wang LJ, et al. Evalution on Clinical Effect of Long Term Shexiang Baoxin Pill Administration for Treatment of Coronary Heart Disease. Chin J Integr Med 2010;30:474-7.

17. Jw L, Wang JL, Qi H. Curative effect observation of Shexiang Baoxin Pill on multi-vessel lesions in CAD without revascularization. Chin $J$ Integr Med 2015;13:1015-516.

18. Yq D. Long-term effect analysis of long term Shexiang Baoxin Pill administration for treatment of coronary heart disease. Guid Chin Med 2016;14:184-5.

19. Zhu $\mathrm{H}$, Luo XP, Wang LJ, et al. Observation on adverse reaction and safety of long term shexiang baoxin pill administration in patients with coronary heart disease. Chin Trad Pat Med 2010;32:2027-8.
20. Zhang YW. Discussion on Adverse Reaction and Safety of Long Term Shexiang Baoxin Pill Administration in Patients with Coronary Heart Disease. Chin J Integr Tradit West Med Cardiovasc Dis 2016;4:163-4.

21. Cardiology Branch of Chinese Medical Association. Editor committee of Chinese journal of cardiology. Chinese guidelines for the prevention and management of chronic stable angina. Chin $J$ Cardio 2007;35:195-204.

22. Cardiology Branch of Chinese Medical Association. Editor committee of Chinese journal of cardiology. Guidelines for the diagnosis and management of non-ST-segment elevation myocardial infarction Acute Coronary Syndromes. Chin J Cardio 2012:40:353-67.

23. Cardiology Branch of Chinese Medical Association. Editor committee of Chinese journal of cardiology. Guidelines for the diagnosis and management of acute ST-segment elevation myocardial infarction. Chin J Cardio 2010;38:675-90.

24. Zheng XY. Guideline of clinical research of new drugs of traditional Chinese medicine: chest obstruction. Chin Med Sci \& Tech Press 2002:68-73.

25. Xie $\mathrm{H}$. Heart of musk pill combined western medicine therapy of coronary heart disease unstable angina random parallel control study. Chin J Pract Int Med 2014;28:105-6.

26. Ly G, Sun ZX. Progress of studies on aspirin resistance and clopidogrel resistance. Chin Hosp Pharm J 2016:866-9.

27. Ma H J YM, Chen $\mathrm{CH}$. Determinants of the medication adherence behavior among elderly patients with coronary heart diseases. J Nurs Educ Pract 2015;5:38-44.

28. Xiong $X$. Integrating traditional Chinese medicine into Western cardiovascular medicine: an evidence-based approach. Nat Rev Cardiol 2015;12:374

29. Xiong XJ, Wang Z, Wang J. Innovative strategy in treating angina pectoris with Chinese patent medicines by promoting blood circulation and removing blood stasis: experience from combination therapy in Chinese medicine. Curr Vasc Pharmacol 2015;13:540-53.

30. Xiong X, Borrelli F, de Sá Ferreira A, et al. Herbal medicines for cardiovascular diseases. Evid Based Complement Alternat Med 2014;2014:1-2

31. Qian W, Xiong X, Fang Z, et al. Protective effect of tetramethylpyrazine on myocardial ischemia-reperfusion injury. Evid Based Complement Alternat Med 2014;2014:1-9.

32. Zhang $\mathrm{H}$, Zhu HY. New interpretation of the advantages of traditional Chinese medicine in the treatment of cardiovascular disease. Chin Arch Tradit Chin Med 2001(03):214-22.

33. Liu HX, Liu P. Clinical characteristic advantage of traditional Chinese medicine in prevention and treatment of cardiovascular disease. Beijing J Tradit Chin Med 2007(07):396-9.

34. Wang J. Current situation and re-understanding of syndrome and formula syndrome in Chinese medicine. Internal Medicine: Open Access 2012;02:e1000113.

35. Wang J, Xiong X. Current Situation and Perspectives of Clinical Study in Integrative Medicine in China. Evidence-Based Complementary and Alternative Medicine 2012;2012:1-11.

36. YL X, WX D. Research summary and consideration of Chinese medicine for the treatment of coronary disease. Heart disease branch of China association of Chinese medicine annual meeting and Beijing Chinese medicine academic society of professional committee on cardiovascular disease 2012:143-5.

37. Chan AW, Tetzlaff JM, Altman DG, et al. SPIRIT 2013 statement: defining standard protocol items for clinical trials. Ann Intern Med 2013;158:200-7.

38. Linde K, Brinkhaus B. Randomized Trials of Chinese Herbal Medicine: A New Extension of the CONSORT Statement. Ann Intern Med 2017;167:133-4. 